\title{
L'entreprise italienne dans la première moitié du XXème siècle. Leopoldo Parodi Delfino : un entrepreneur éclectique
}

\section{Italian entrepreneurship in the first half of the 20th century. Leopoldo} Parodi Delfino: an eclectic entrepreneur

\author{
Nardone Paola ${ }^{1}$, Ridolfi Natascia ${ }^{2}$, Di Nucci Ada $^{3}$ \\ ${ }^{1}$ Département de Science Philosophique, Pédagogique, Economique-Quantitative, Université de Chieti-Pescara, \\ Italie, paola.nardone@unich.it \\ ${ }^{2}$ Département de Science Philosophique, Pédagogique, Economique-Quantitative, Université de Chieti-Pescara, \\ Italie, natascia.ridolfi@unich.it \\ ${ }^{3}$ Département de Science Philosophique, Pédagogique, Economique-Quantitative, Université de Chieti-Pescara, \\ Italie, a.dinucci@unich.it
}

RÉSUMÉ. Dans la première moitié du $X X^{e}$ siècle l'industrie italienne se renforce et assume les caractéristiques de la grande entreprise. Parmi les protagonistes de cette période le rôle de l'ingénieur Leopoldo Parodi Delfino se révèle, une figure éclectique qui commence son activité dans le secteur des liqueurs et du vin, constituant en 1902 la « Società Fabbrica Nazionale Alcool Leopoldo Parodi Delfino »; quelques années plus tard il créa la " Distilleria Nazionale » par laquelle il réussit à acquérir les sociétés « Distillerie Italiane » et les «Stabilimenti Vinicoli Florio ». En 1912 il passa à la production des explosifs, créant avec l'entrepreneur Giovanni Bombrini la "Società in nome collettivo Bombrini Parodi Delfino ». En 1918 il participa à la constitution de la "Società Mediterranea di Elettricità ». Ses capacités de gestion et sa position financière lui ont même permis d'entreprendre d'importants investissements à l'étranger. En 1918, il reprend la "Société des Mines de Selenitza » en Albanie et en 1921 il constitue en Equateur la " Compagnia italiana dell'Equatore ». En 1939 il est nommé Sénateur du Royaume d'Italie.

ABSTRACT. In the first half of the 20th century the Italian industry was reinforced and took the features of the high enterprise. Among the protagonists of this period was the engineer Leopoldo Parodi Delfino, a very eclectic figure who started his own activity in the liqueur and wine sectors, creating in 1902 the "Società Fabbrica Nazionale Alcool Leopoldo Parodi Delfino"; some years later he built the "Distilleria Nazionale" with which he succeeded in buying the companies "Distillerie Italiane" and the "Stabilimenti vinicoli Florio". In 1912 he moved over to explosives production, creating together with the entrepreneur Giovanni Bombrini the "Società in nome collettivo Bombrini Parodi Delfino". In 1918 he participated in the constitution of the "Società Mediterranea di Elettricità". His management abilities and his financial position permitted him to also make some huge investments in foreign countries. In 1918 he effectively took over the "Société des Mines de Selenitza" in Albania, and in 1921 he built the "Compagnia italiana dell'Equatore" in Ecuador. In 1939 he was appointed Senator of the Kingdom of Italy.

MOTS-CLÉS. Entrepreneur, innovateur, stratégie, marchés, développement, ingénieur, chimie, explosifs.

KEYWORDS. Entrepreneur, innovator, strategy, markets, development, engineer, chemistry, explosives.

\section{Introduction}

Entre 1897 et 1907 l'Italie a bénéficié d'une forte expansion industrielle : le PIB total et par habitant ont augmenté respectivement du 2,4\% et du 1,6\% par an [TON 13]. Le taux de croissance moyen de la production industrielle est passé du 0,3\% au 6,7\% [PEC 05], par contre la production agricole n'a augmenté que de $1,7 \%$. Au cours de cette période l'État commence à combler le fort écart qui se crée au niveau économique avec les États-Unis, l'Allemagne, la France et l'Angleterre, en soutenant le développement de la grande entreprise, des infrastructures et des communications, ainsi que l'assainissement des comptes publics [PEC 05]. Le tissu industriel italien s'articulait autour de deux noyaux : le premier typique de l'industrie lourde est caractérisé par des établissements de grandes dimensions dotés d'installations technologiques qui nécessitaient des capitaux considérables et d'une intense activité de recherche scientifique, axés sur l'innovation et 
sur des marchés en extension; le second noyau, incluant les petites et moyennes entreprises (qui ensuite caractérisa le modèle de développement national des districts industriels), était lié à la grande production soit à travers les approvisionnements de produits semi-finis et matériaux associés, soit en approvisionnant les services de support aux grandes entreprises. Les plus grands établissements industriels étaient situés surtout dans le « triangle » Milan-Turin-Gênes qui concentrait 55\% du revenu national du pays en 1911 [TON 13].

La plupart des entreprises industrielles italiennes se concentraient dans les secteurs de chimie, du matériel électrique, du pétrole, de la métallurgie, la mécanique et des moyens de transports. Les entrepreneurs avaient adopté des stratégies visant à privilégier la diversification de la production et l'augmentation de leurs capacités productives, en réduisant les coûts unitaires et en réalisant des économies d'échelle. Dans ce but, atteindre un haut niveau d'intégration verticale autant en amont qu'en aval était indispensable, ceci en triplant les investissements dans l'aménagement productif, la distribution et le management [AMA 11]. Le développement industriel favorisa la montée en puissance d'une nouvelle catégorie d'entrepreneurs, les capitaines d'industrie, ouverts à l'innovation et à l'expansion commerciale vers les marchés étrangers. Ces caractéristiques incarnaient le modèle de l'entrepreneur qui, quelques années plus tard, a été théorisé par Joseph Alois Schumpeter [SAN 84] [SCH 11]. Ces nouveaux acteurs étaient stimulés par :

« proposito, ora reso sempre più esplicito, di imporre il sistema industriale come modello alternativo alla vecchia società rurale... sulla base dei principi propri dello sviluppo capitalistico: l'etica del profitto, l'individualismo, un gusto pratico per le innovazioni, la convinzione che la fabbrica fosse il perno e la sede strategica dell'accumulazione economica e delle trasformazioni sociali » [CAS 95].

(« l'objectif, devenu maintenant toujours plus clair, d'imposer le système industriel comme modèle alternatif par rapport à la vieille société rurale... sur la base des seuls principes du développement capitaliste : l'éthique du profit, l'individualisme, un goût pratique pour les innovations, la conviction que l'usine représente l'axe et le siège stratégique de l'accumulation économique et des transformations sociales »).

Cette classe montante d'entrepreneurs maitrisait une culture entrepreneuriale moderne, acquise la plupart du temps au cours d'études à l'étranger. Elle réalisa des choix stratégiques courageux capables de créer de la richesse sans bouleverser le système de l'usine. Un « groupe hétérogène par le statut social et par les parcours de formation » émergea. Il s'orientait vers une transformation de la vision traditionnelle de l'entrepreneur et de l'entreprise [BES 84]. Cette catégorie était composée d'innovateurs, d'héritiers de familles ouvrières qui avaient fait des études secondaires (par exemple Edoardo Bianchi et Ercole Marelli) et des entrepreneurs qui provenaient par contre de classes aisées ayant suivi un parcours universitaire (par exemple Giovanni Agnelli, Artuto Gazzoni, Camillo Olivetti, Vincenzo Lancia) [SCA 11]. Ils se rapprochaient pour leurs conceptions dynamiques et vivantes de la direction d'entreprise, caractérisée par des investissements destinés au « lancement des produits, à l'introduction de nouvelles méthodes de fabrication, et à la conquête de nouveaux marchés »[DOR 84]. Ils étaient les vrais acteurs de la vie économique, les seuls capables d'entreprendre des actions « grandioses », qui les élevaient au-dessus des autres acteurs économiques. Cette nouvelle classe d'entrepreneurs n'avait aucune crainte d'assumer les risques liés aux activités et elle avait la capacité de faire face aux incertitudes au nom du profit. C'est dans cette ambiance que se formèrent la personnalité et le parcours de l'ingénieur Leopoldo Parodi Delfino, acteur de l'économie italienne dans la première moitié du vingtième siècle. Il incarna le modèle de l'entrepreneur innovateur, dont l'activité marqua fortement le contexte économique et industriel de la période.

L'objectif de l'article est de reconstruire, à travers les archives, la personnalité et le parcours de l'entrepreneur Leopoldo Parodi Delfino. Les documents consultés sont ceux qui ont été déposés auprès des Archives de la Fondazione Ansaldo de Gênes, le fonds des Archives Historiques de la 
Municipalité de Colleferro, celui de la Banca Intesa de Milan, les Archives Centrales de l'État et les Archives Historiques du Ministère des Affaires Etrangères tous deux situés à Rome. Nous présenterons d'abord les phases qui ont caractérisé l'évolution de son activité d'entrepreneur, qui commença par l'achat des premières usines et continua vers la constitution de la société Bombrini Parodi Delfino et la construction du village industriel de Colleferro. Nous nous pencherons ensuite sur les innovations technologiques que l'entrepreneur développa avec succès dans les entreprises qu'il créa.

\section{L'ascension de l'entrepreneur}

Leopoldo Parodi Delfino est né à Milan en 1875 [TOS 19] ${ }^{1}$. Après avoir obtenu son baccalauréat en ingénierie chimique à l'École polytechnique fédérale de Zurich, il perfectionna sa formation à 1'Université de Leipzig et à celle de Breslau (aujourd'hui Wroclaw) ${ }^{2}$. Il commença à travailler dans la distillerie familiale, dans le Piémont, il en prit bien vite sa direction. Cette expérience a été fondamentale pour sa connaissance du monde de l'entreprise. En 1902, à seulement vingt-sept ans, il commença sa propre activité en créant la Società Fabbrica Nazionale Alcool Leopoldo ParodiDelfino [LAC 46] dont le siège fut installé à Savone, près du port de Gênes, un des points principaux du commerce national et international et, donc, stratégique pour la vente de ses produits. Deux années plus tard, en 1904, il créa la Distilleria Nazionale à Pontelagoscuro, sur les rives du Po, dans la province de Ferrare. Elle fut la première usine italienne à produire de l'alcool éthylique neutre à partir de la mélasse de betterave, une innovation technologique de premier plan, qui permit d'abaisser drastiquement le prix en raison des taxes d'importation de l'alcool. Le succès obtenu dans ce secteur le conduisit à étendre son activité entrepreneuriale avec l'acquisition de deux sociétés : en 1905 il acheta la Distillerie Italiane, à Milan, financée par la Banca Commerciale, par le Credito Italiano et par la Società Bancaria Italiana ; en 1904 il siégeait dans le conseil de la SAVI (Società Anonima Vinicola Italiana Florio \& C.), dont les parts étaient détenues par la Banca Commerciale Italiana ${ }^{3}$ [CAN 08], qui passa sous le contrôle des Distillerie Italiane en 1907 [DAT 94].

Après avoir renforcé ses compétences managériales Parodi Delfino put élargir ses activités en se tournant vers l'industrie lourde et la chimie, objet de sa formation scientifique universitaire. L'occasion se présenta entre 1902 et 1906 après l'adoption d'une série de lois en faveur des zones défavorisées du pays. Parmi les travaux à réaliser, se trouvait l'aqueduc de la Puglia, un des plus grands du monde, pour lequel de très importantes subventions publiques avaient été allouées. La construction de l'ouvrage stimula l'intérêt de quelques entrepreneurs de Gênes qui, comptant sur le tissu dual des entreprises métallurgiques de la Ligurie, décidèrent d'investir dans les infrastructures du sud de l'Italie.

Parodi Delfino s'associa à un groupe d'entrepreneurs, dont Giovanni Bombrini et Ferdinando Maria Perrone (de la Società Gio. Ansaldo \& C.) [CAS 94] [HER 96] [CAS 97] qui avec la Società anonima Ercoli Antico e soci ${ }^{4}$ [QUI 15], obtint le marché pour la construction de l'aqueduc ${ }^{5}$. Les

\footnotetext{
${ }^{1}$ Son père Carlo Giuseppe Delfino et sa mère Marina Parodi étaient les rejetons de l'élite des commerçants et des banquiers italiens. Leopoldo avait quatre frères et une sœur.

${ }^{2}$ II Popolo d’Italia,10 août 1939.

${ }^{3}$ L'entrepreneur Ignazio Florio a été forcé à céder, en garantie de ces propres dettes, outre 30.000 actions de la prestigieuse entreprise.

${ }^{4}$ En réalité la société est née le 19 mars 1904 comme Ditta Ercole Antico et Soci, dont le siège était à Genova et le modeste capital de 1.515.000 lire. Après trois mois de la victoire dans l'appel d'offres elle se transforma en Società Anonima Ercole Antico, déplaçant son siège à Roma.
}

${ }^{5}$ Decreto Ministeriale (Décret Ministériel) du 6 août 1905, Fondo Acquedotto Pugliese SpA (Fond Acqueduc de la Puglia SpA). 
travaux commencèrent en 1906, mais subirent tout de suite un ralentissement ${ }^{6}$ dû à la complète inexpérience de la société dans la construction d'un ouvrage aussi colossal et au fait qu'elle disposait d'un capital très limité. Elle ne pouvait pas poursuivre les travaux sans obtenir une contribution de l'État [QUI 15] $]^{7}$. Il s'agissait d'un pari financier très important alors que la crise économique internationale de 1907 toucha profondément le système bancaire et financier italien, occasionnant un coup d'arrêt au développement économique. En 1911, le pays fut lourdement touché par une épidémie de choléra. La société Ercole Antico accumula des dettes et un irrémédiable retard sur le calendrier des travaux, il devint évident que le respect des délais prescrits (décembre 1914) pour réaliser l'ouvrage était impossible à tenir. Le gouvernement central appliqua à l'entreprise tributaire du marché de lourdes pénalités qui firent empirer la situation [BAR 86], puis le début de la première Guerre Mondiale mis fin aux travaux, une nouvelle société lui succéda en 1919, l'établissement Autonomo pour l'Aquedotto Pugliese (EAAP), qui racheta la totalité de l'installation et reprit ses dettes ${ }^{8}$.

Ce contexte difficile n'atteint pas sérieusement les anciennes activités de distillation de Parodi Delfino. Au contraire, l'ingénieur réalisa très rapidement de nouveaux projets. En 1909 il prit part à la création de la Società Smalteria Italiane, de Milan, dont il devint président. Sous sa direction, la société impulsa un «notable élan au secteur entier » du fer émaillé 9 .

Le succès, les capacités scientifiques, professionnelles, managériales et techniques de Parodi Delfino éveillèrent l'intérêt des hauts responsables de l'État : l'ingénieur fut alors directement contacté et impliqué par le chef du gouvernement, Giovanni Giolitti, dans le projet de renforcement technologique de l'industrie militaire nationale. Pendant ces années, la situation politique européenne était sous tension et les prévisions d'un conflit imminent préoccupaient largement les gouvernements européens, en particulier l'Italie qui, du point de vue de l'industrie de l'armement, était en retard, en raison une capacité productive très limitée et obsolète. Très peu d'usines étaient dédiées à la production des explosifs, parmi celles-ci on comptait l'usine de poudres explosives de Avignana, celle de Spilamberto (Modène) et l'usine royale de poudre explosive de Fontana Liri Inferiore $^{10}$. Les industries militaires se trouvaient à Terni, Pozzuoli, La Spezia et Gênes. En 1912, le gouvernement italien demanda à Parodi Delfino et au sénateur Giovanni Bombrini, propriétaire de la Société Ansaldo de Gênes et déjà associés dans l'expérience de l'aqueduc de la Puglia, de créer la Société en nom collectif Bombrini Parodi Delfino (BPD) dont le siège social serait à Rome, avec l'objectif de produire et d'élaborer des explosifs, un programme complexe dans lequel de nombreuses ressources et capitaux furent engagés. Pour réaliser ce projet, un grand établissement fut construit sur le site indiqué par l'ingénieur ${ }^{11}$, près du village de Segni Scalo, (actuelle Colleferro dans la province de Rome), où l'activité commença grâce à l'aide de techniciens du Piémont,

\footnotetext{
${ }^{6}$ Archive Historique Ansaldo (ASA), Archive Parodi Delfino (APD), Acquedotto Pugliese.

${ }^{7}$ La loi prévoyait l'annulation des financements a posteriori, et non pas pour des sommes supérieures aux dépenses soutenues. L'immobilisation aurait été remboursée avec les entrées de l'activité même, attribuée à la Concessionaria pour 90 ans. Une clause qui aurait dû faire office de levier pour la rapidité des travaux.

${ }^{8}$ Décret Royal Législatif du 10 avril 1919.

9 « Necrologio di Leopoldo Parodi-Delfino », La chimica e l'industria, Milano, n 1-2, p. 27, 1946.

${ }^{10}$ Archive Historique Mairie de Colleferro (ASCColleferro), Archive Bombrini-Parodi Delfino (BPD), Colleferro, Relazione Tito Benelli, Origine e sviluppo degli stabilimenti di Colleferro, octobre 1912-octobre 1937.

${ }^{11}$ Sur les bases d'une vieille usine de sucre de la société Valsacco située sur le terrain acheté par la BPD. ASCColleferro, BPD, Relazione del dottore Aldo Colajacono, Origini dello stabilimento BPD di Colleferro, 24 février 1966, p. 10.
} 
experts des explosifs ${ }^{12}$. En peu d'années, la société se plaça au premier plan de l'industrie chimique nationale et internationale.

L'expérimentation de nouvelles technologies continua en août 1918 et, vers la fin du conflit mondial, elle participa à la constitution de la Società Mediterranea di Elettricità. En s'unissant à la BPD, elle prit part à Milan, avec la Banca Commerciale Italiana et la Banca Zaccaria Pisa, à la création de la société anonyme Soi de Châtillon, un établissement construit dans une municipalité anonyme du Val d'Aoste, en une position stratégique, près des forêts et des centrales hydroélectriques. Le but social était de produire de la viscose, une nouvelle fibre qui était en train de prendre place avec succès dans le marché. L'ingénieur prit part à la direction de cette société pendant les huit premières années et, sous sa direction, l'entreprise s'agrandit avec un nouvel établissement à Ivrée et un autre à Vercelli. En 1924, Parodi Delfino possédait déjà 30\% des actions de la société [TOS 02].

Pendant la première partie de l'après-guerre, Parodi Delfino était pleinement introduit parmi les savants et les dirigeants d'entreprise italiens au service de la nation. Conforté par ses expériences professionnelles, il était prêt à développer son activité au-delà des frontières. Dans la phase de renouvellement social et d'expansion économique que connaissait l'Italie, le gouvernement incitait les entrepreneurs à investir dans le nouveau protectorat albanais, pour consolider la présence italienne sur place. Sous les pressions conjointes des Ministères de la Marine et de l'Étranger, l'ingénieur intervint dans la zone de Valona pour y exploiter des mines bitumeuses et lança une activité de prospection pétrolière avec la Société des mines de Selenizza, un grand établissement dédié au traitement du goudron et à la production des huiles minérales, construit en 1918 [DAM 80]. Bientôt la société devint leader international du secteur des bitumes, goudrons et asphaltes [ORL 11]. En 1922, elle employait plus de 500 travailleurs. De 1920 à 1943, sous la direction de Parodi Delfino, la production atteignit environ les 20.000 tonnes annuelles de bitume.

Le gouvernement italien voulait également développer son action en direction de l'Equateur. Parodi Delfino fut de nouveau sollicité. Avec sa société BPD, il créa en 1921 la CIDE (Compagnia Italiana dell'Equatore) ${ }^{13}$ avec l'objectif d'exercer une forte influence financière sur le développement économique du pays $^{14}$. Le projet était ambitieux car l'Equateur était fortement endetté et, pour cette raison, contrôlé par les États-Unis qui tentèrent de s'opposer à l'influence italienne dans cet état sud-américain [SOA 08]. La CIDE agissait sous la tutelle du gouvernement italien et incarnait l'intérêt national à l'étranger. Le programme qui lui fut confié était de créer une banque, des établissements agricoles et commerciaux, dans les travaux publics ainsi que dans l'exploitation des ressources naturelles ${ }^{15}$. En 1921, l'ingénieur envoya sur place, dans un premier temps pour un contrôle, une trentaine de techniciens de la BPD qui élaborèrent un programme d'investissement et de modernisation des infrastructures du pays, qui devait limiter ses dépenses et augmenter les impôts ; l'Italie devait recevoir quelques monopoles dans l'exploitation minière et dans la colonisation agricole [SOA 08]. Une Banque Italienne de Guayaquil, dont Leopoldo devint président, fut créé dans ce but. Elle s'ingéra fortement dans le système bancaire national, en émettant de la monnaie pour financer des projets nationaux. À travers plusieurs sociétés, elle réalisa des bâtiments publics, parmi lesquels le palais du gouvernement, la mairie, l'hôpital, et des lieux de culte, dont la cathédrale centrale et des églises de périphérie, et aussi des immeubles destinés aux

\footnotetext{
${ }^{12}$ https://cittamorandiana.it/la-nascita-della-bpd/ (accessed 11/06/2020).

13 Archive Historique Banca Intesa (ASI), Banca Commerciale Italiana (BCI), Ufficio finanziario, Pratiche della Segreteria finanziaria, Milano, 14 juin 1929.

${ }^{14} \mathrm{ASI}, \mathrm{BCl}$, Ufficio finanziario, Pratiche della Segreteria finanziaria, 21, 4, Milano, 14 juin 1929. Dans ce projet il y avait aussi un engagement de la Banca Italiana di Sconto, de la Società Agricola Commerciale Industriale et la Banca Commerciale

${ }^{15} \mathrm{ASI}, \mathrm{BCl}$, Verbale del Consiglio di amministrazione, Vol. 6, p. 41, 22 octobre 1920.
} 
activités économiques et commerciales comme l'édifice de la Previsora [SOA 08]. Malheureusement la création d'une entreprise de travaux publics qui devait coordonner l'exploitation des ressources naturelles et les concessions obtenues du gouvernement équatorien échoua en raison de son coût élevé, des réticences politiques des gouvernements italien et équatorien et de l'ingérence des États-Unis ${ }^{16}$ [TOS 02]. La CIDE fut dissoute à la veille de la Seconde Guerre mondiale dans un contexte politique et économique qui imposait à l'Italie de retrouver ses forces et de rapatrier ses capitaux vers la mère-patrie en prévision de son entrée en guerre ${ }^{17}$.

A partir des années 1920, la vie politique italienne fut en ébullition avec l'ascension du nouveau parti national fasciste. Parodi Delfino, comme du reste d'autres industriels de cette période, s'en rapprochèrent avant la marche sur Rome (1922). Ceux-ci espéraient créer un lien économique entre le fascisme et la classe industrielle, à laquelle le parti avait promis le contrôle et la direction de l'économie du pays que celle-ci désirait, surtout après les violentes manifestations ouvrières contre les propriétaires et les entrepreneurs. Parodi Delfino s'adapta à ce nouveau contexte au nom de son activité et de ses projets entrepreneuriaux. En 1932, il adhéra au parti fasciste qui en apprécia immédiatement les talents scientifiques et professionnels. Il devint une référence au sein de la Fédération nationale fasciste des Industriels de la chimie. De nombreuses reconnaissances et charges lui furent attribuées : en 1932 il fut nommé président de la section Industrielle des Conseils provinciaux des Corporations de Rome. En 1937 il fut nommé Chevalier de l'ordre du Travail, puis en 1939 il devint sénateur (inscrit à l'Union Nationale Fasciste du Sénat). Il était aussi engagé dans une série de comités : celui de la Finance, celui de l'Économie corporative et de l'autarcie et, enfin, celui des Forces armées. Il gardait cependant sa lucidité s'éloignant des idées du fascisme ${ }^{18}$. Sa position intransigeante, dans les affaires et dans la politique, lui conférèrent le surnom de « Sénateur de Fer » [MAR 83]. Il s'adapta avec habilité au pouvoir politique, conservant toujours une opinion et une action qui ne s'opposaient pas directement au régime, s'assurant ainsi une relative et confortable « liberté». Cette approbation «physiologique » du fascisme l'engageait personnellement, tout comme les autres entrepreneurs de cette période [BAR 18] [MES 17] [FAT 16]. D'une certaine façon, il dirigea son entreprise pour recevoir des commandes d'État et conserver ses activités et ses ouvriers [ORL 11]. Probablement s'adapta-t-il au contexte politique pour des raisons économiques et par rapport aux industriels de l'époque, accepta-t-il les règles du régime dans un rapport forcé ? Toutefois, il en tirait avantage car l'industrie des explosifs satisfaisait les attentes du gouvernement qui planifiait l'organisation armée du pays. Après la chute du fascisme, Parodi Delfino se plaça du côté du mouvement des partisans. Suite au débarquement des Alliés à Anzio il participa à la libération du pays en fournissant des armes et des munitions, produites illégalement par l'usine de Colleferro ${ }^{19}$.

Toutefois à la fin de la guerre, l'ingénieur fut accusé par la Haute Cour de Justice pour avoir favorisé le régime fasciste. Au cours de son procès il réfuta toutes les accusations contre lui en soutenant, entre autres, que la plupart de ses entreprises furent créées avant l'instauration du régime ${ }^{20}$ [CAN 99]. Sa position ne fut reconnue qu'après sa mort, survenue le 13 novembre 1945.

\footnotetext{
${ }^{16}$ Sur Banco italiano de Guayaquil voir: Archive Historique Ministère des Affaires de l'Étranger (ASMAE), Affaires Politiques, 1919-1930, Equatore, 1013, 2844, Programma per una penetrazione economica italiana nell'Equatore.

${ }^{17} \mathrm{ASI}, \mathrm{BCl}$, Bureau des Finances, Pratiche della Segreteria finanziaria, 21, 4, Extrait des délibérations Assemblée ordinaire et extraordinaire, 17 juin 1936.

${ }^{18}$ Archive Sénat du Reigne, Resoconti delle discussioni, XXX legislatura, 1 de la Chambre des Faisceaux et des Corporations, Année 1940 (XVIII-XIX) Volume deux, Imprimerie du Sénat, Roma, p. 126, 1941 - XIX.

${ }^{19}$ Archive Sénat de la République, Relazione dell'Alta Corte di Giustizia per le sanzioni contro il fascismo, 22 février 1943.

${ }^{20}$ Archive Central de l’État, Épuration Sénat, Mémoires défensives, mémoire du 20 janvier 1947.
} 


\subsection{Leopoldo Parodi Delfino : le pionnier des explosifs dans I'Industrie de Colleferro}

L'ingénieur renforça sa position dans l'entreprise et dans les finances grâce à la société Bombrini Parodi Delfino, où il appliqua une stratégie d'entreprise tournée vers l'innovation. Bien avant la création de celle-ci, Parodi avait bien compris que l'affaire que Giovanni Giolitti lui avait offerte (en février 1912) présentait l'avantage d'une absence de concurrents et d'un retour sur investissement dans un temps très réduit.

Le gouvernement Giolitti voulait :

«provvedere autonomamente a creare con capitali e gestione esclusivamente italiani un polverificio moderno, efficiente, capace di produrre la quantità di balistite per cannoni, di dinamite per mina e di munizioni per artiglieria necessaria a un'eventuale guerra »[ORL 11].

(« assurer de façon autonome la création, à travers des capitaux et une gestion exclusivement italiens, d'une usine de poudre moderne, performante, capable de produire la quantité de balistite pour canons, de dynamite pour les mines et des munitions pour l'artillerie nécessaires à une possible guerre »).

De cette façon, l'État aurait géré l'offre de matériels militaires à l'intérieur du pays en finançant directement la production et en stimulant l'évolution de l'industrie nationale, afin d'éviter des financements qui auraient pu renforcer la position des industries militaires étrangères. C'est ainsi qu'entre 1905 et 1913 le gouvernement finança, pour les productions nationales, environ 140 millions de lires, limitant les achats au-delà des frontières à environ 2 millions de lires [LIZ 11].

Le seul point faible du projet de Giolitti se trouvait dans le temps limité de réalisation. Pour cette raison, Parodi Delfino s'associa à Giovanni Bombrini, un partenaire financier parfait car il était déjà un entrepreneur de prestige, et aussi sénateur du royaume d'Italie, ce qui pouvait faciliter toutes les procédures administratives ${ }^{21}$. Un dernier point en faveur de Bombrini était l'intense esprit de nationalisme de cette période : la production de guerre ne pouvait certainement pas être partagée avec un partenaire étranger et les entrepreneurs concernés devaient être « exclusivement des personnes italiennes, absolument pas liés à des groupes étrangers qui pourraient éventuellement être en conflit avec notre pays $»^{22}$.

Bombrini et Parodi Delfino créèrent une société en nom collectif en Octobre 1912, forme juridique qui permettait d'avoir sous contrôle la propriété du capital social et d'éviter la fuite d'informations qui auraient $\mathrm{pu}$ intéresser la concurrence ${ }^{23}$. Peu après, ils réalisèrent un grand établissement à Colleferro ${ }^{24}$, un lieu stratégique loin de la côte et donc d'éventuelles attaques provenant de la mer, mais près de Rome, centre du pouvoir et des intérêts économiques, et sur le fleuve Sacco, dont les eaux pouvaient être exploitées pour la fabrication des explosifs, tandis que la proximité d'un embranchement ferroviaire facilitait des transports ${ }^{25}$. L'usine n'était pas loin de la poudrerie royale de Fontana Liri Inferiore, avec laquelle Parodi Delfino avait l'intention de nouer des relations de collaboration et d'aide mutuelle en cas de nécessité ${ }^{26}$. De plus, les régions en

\footnotetext{
${ }^{21}$ ASCColleferro, BPD, Relazione del dottore Aldo Colajacono, Origini dello stabilimento BPD di Colleferro, 24 février 1966, p. 10.

${ }^{22}$ ASA, Société Bombrini Parodi Delfino (SBPD), cc. 210, 1912-1936, Relazione Leopoldo Parodi Delfino, juin 1912, c. 2.

${ }^{23}$ Ibidem.

${ }^{24}$ Ivi, c. 3.

${ }^{25}$ ASCColleferro, BPD, 25 ans de vie de la B.P.D., Relazione del dottore Tito Benelli, Directeur de l'usine, octobre 1912-octobre 1937.

${ }^{26}$ Ibidem. 
développement pouvaient offrir des marges de croissance bien supérieures par rapport aux territoires déjà industrialisés. Elles représentaient le milieu idéal pour accueillir les nouvelles structures industrielles capables d'accueillir le savoir-faire et les politiques industrielles innovantes. Le choix était donc raisonné et stratégique dans la vision de l'entrepreneur Leopoldo Parodo Delfino.

Dans l'usine de Colleferro, il décida personnellement de la mise en place de chaque machine. Dans la phase d'installation, il dirigea une équipe de techniciens de l'industrie chimique, pour concevoir la ligne de production à installer. Il fallait définir les marchés pour la fourniture d'une part des matières premières comme le benzol, le toluène, la glycérine, d'autre part des réactifs comme l'acide nitrique et l'acide sulfurique [MOL 32]. Parodi Delfino décida que le coût de la nitroglycérine, y compris les frais de main-d'œuvre, ne devait dépasser les 2 lires au $\mathrm{kg}^{27}$. Dès lors, il détermina la quantité des installations pour la production des explosifs. La BPD nécessitait une « installation pour la préparation de la nitroglycérine » et une autre «installation pour le mélange de cette marchandise et pour la préparation des cartouches $»^{28}$. Par contre, pour la production de la « dynamite I » (dynamite première, c'est-à-dire à base inerte) [GRO 31], il fallait construire une usine pour « mélanger $60 \%$ de [dynamite] caoutchouc $30 \%$ de nitrate de soude et $10 \%$ de sciure de bois moulu ${ }^{29}$. Selon ces prévisions, la production de l'établissement, selon l'ingénieur, pouvait atteindre quotidiennement les « $1000 \mathrm{~kg}$ de balistite (chiffres redoublés par un travail de 24 heures au lieu de 12) et $1000 \mathrm{~kg}$ de dynamite gomme $»^{30}$. Parodi Delfino accorda aussi une attention particulière aux techniques de production et, dès décembre 1912, il organisa une série de rencontres avec des experts internationaux du secteur, pour évaluer les innovations à introduire sur les lignes de fabrication de l'établissement de Colleferro. Il se mit en contact avec les plus grands représentants industriels et scientifiques au niveau international et, lors d'une rencontre à Zurich, il obtint les informations nécessaires pour produire l'acide nitrique, pour dénitrer les mélanges acides mais, aussi, pour concentrer et faire converger en une seule zone l'acide sulfurique. Les informations obtenues du professeur de chimie technologique Giorgio Lunge [AET 28] du Politecnico Federale Elvetico, sur le processus de nitration et l'essorage par la méthode Selwig et Lange ${ }^{31}$, ainsi que la connaissance du système de stabilisation de la nitrocellulose conçu par Voith ${ }^{32}$, ont été très importantes pour développer la production de l'établissement de Colleferro. Parodi Delfino montra aussi un intérêt pour le système de Uebel et de Valentiner relatifs à la fabrication de l'acide nitrique et celui de la production d'acide sulfurique à travers la méthode de Gaillard [DEF 28]. Toutes deux furent introduites dans l'établissement BPD.

Enfin, Parodi Delfino intervint personnellement dans la recherche et le développement des machines et d'équipements à l'avant-garde. A cette fin, il visita l'établissement allemand de Friedrich Krupp, à Magdeburg, où il apprit puis expérimenta pour la première fois en Italie, une série d'innovations, tels que l'emploi de pressoirs spéciaux pour la production des poudres à la phtalide sans l'utilisation d'un solvant, la production de la poudre calibre 12 et celle pour les cartouches des mitrailleuses lourdes, de type 13,2 $2^{33}$. En Allemagne, à Geislingen an der Steige, il acheta des machines pour la fabrication des poudres absorbantes pour la dynamite. Ensuite il se

\footnotetext{
${ }^{27}$ ASA, SBPD, cc. 210, 1912-1936, Relazione Leopoldo Parodi Delfino, juin 1912, c. 3.

${ }^{28}$ Ivi, c. 2.

${ }^{29}$ Ibidem.

${ }^{30}$ ASCColleferro, BPD, 25 ans de vie de la B. P. D., Relazione del dottore Tito Benelli, Directeur de l'usine, octobre 1912-octobre 1937, p. 3.

${ }^{31}$ Ivi, p. 4.

32 Ibidem

${ }^{33}$ Ivi, p. 5.
} 
rendit en Belgique où il analysa la méthode de purification et distillation de la glycérine et, enfin, il alla en Angleterre à Waltham Abbey où il obtint le brevet pour la fabrication de la nitroglycérine, développée pour la Royal Gunpowder Factory chez F. L. Nathan, J. M. Thompson et W. Rintoul [MAR 12]. Le dispositif était constitué d'un récipient de plomb cylindrique avec un fond incliné contenant des bobines de refroidissement qui employaient l'ammoniaque comme réfrigérant et des tubes d'air pour le processus de brassage. Quand il revint en Italie, Parodi Delfino apporta des modifications à ses installations : il intensifia et améliora le récipient de plomb cylindrique utilisé pour la production de la nitroglycérine et, pour la fabrication des acides, il employa des réservoirs et des chaudières soudées sans rivets ${ }^{34}$.

En 1913, après ces visites dans les plus grandes industries chimiques d'Europe et de nombreux échanges avec les plus importants experts internationaux, Parodi Delfino estima avoir acquis une vision complète des systèmes et des techniques de production du secteur. Ces expériences étrangères ont été très importantes pour donner une nouvelle impulsion à son entreprise. L'entrepreneurinnovateur ne se posait aucune limite géographique afin d'acquérir des technologies d'avant-garde qui n'existaient pas sur le territoire italien [ORL 11]. Fin 1913, la BPD engageait la production d'acide nitrique et la «purification et distillation de la glycérine », puis dans la première moitié de 1914, il commença la production de la dynamite et de la balistite pour l'armée italienne ${ }^{35}$.

Avec l'entrée en guerre de l'Italie, l'usine commença à travailler à plein-temps, avec une production en cycle continu, sans interruption. La guerre augmenta le niveau des affaires et la stratégie de l'entreprise s'adapta immédiatement au contexte du conflit : la BPD renforça son usine et introduisit des nouvelles lignes de machines qui arrivèrent à produire 22 tonnes par jour de balistite et 5 tonnes de dynamite.

La guerre poussa le gouvernement italien à demander aux entrepreneurs du secteur d'inventer et de réaliser de nouvelles armes destinées aux Forces Armées. C'est ainsi que l'établissement de Colleferro commença la production de grenades à main de «type spécial » chargées avec des « des morceaux de balistite et un explosif appelé B.P.D. $»^{36}$ (du nom de la société), « grenades de type 140,162 et 260 » et des «balles incendiaires » pour lesquelles on préparait chaque jour des tonneaux de pulvérins destinés aux déclencheurs. En 1918, l'industrie était prête pour la production du $\mathrm{TNT}^{37}$.

A la fin de la Première Guerre mondiale, la BPD était une usine à l'avant-garde. Les installations qui réalisaient des explosifs

« rappresentarono dei modelli di versatilità e di capacità produttiva, si trattava di un sistema di fabbriche di alta organicità ed efficienza, avente un'unica direzione centralizzata, che coordinava la corretta esecuzione di una larga complessità di tecnologie industriali »[ORL 11].

(« représentèrent des modèles de versatilité et de capacité productive, il s'agissait d'un système d'usines de haute cohérence et rendement, ayant un seul but centralisé, qui coordonnait l'exécution parfaite d'une ample complexité de technologies industrielles »).

Parodi Delfino convertit la production vers les activités de paix. Les « explosifs de lancement », développés pour la guerre, furent utilisés pour défricher les terrains, extirper les arbres et augmenter

\footnotetext{
${ }^{34}$ Ibidem.

${ }^{35}$ ASCColleferro, BPD, 25 ans de vie de la B. P. D., Relazione del dottore Tito Benelli, Directeur de l'usine, octobre 1912-octobre 1937, p. 5.

${ }^{36}$ Ivi, p. 6. 
la surface agricole utilisable pour la culture du blé. De nouvelles productions commencèrent : en 1919, les engrais phosphatés et en 1920 le béton. La reconstruction de l'après-guerre nécessitait d'immenses quantités de béton et de ciment et bientôt l'établissement de Colleferro ne réussit plus à satisfaire la demande du marché, il fallut construire une usine dédiée. L'ingénieur décida de diversifier la production de la BPD en créant en 1921 la Società Produzione Calce e Cementi di Segni. Le béton fabriqué était de nouvelle génération : un « ciment béton » aux caractéristiques innovantes, de très haute qualité et résistance [TOS 02] [ILG 62]. Encore une fois, l'intuition de l'entrepreneur Parodi Delfino se confirma avec succès. La production de ciment atteignit en deux ans les 700 tonnes par jour [TOS 02], elle fut accompagnée par la chaux hydratée, produite en substitution de la chaux vive avec d'évidents avantages par rapport à son emploi, à sa performance et à son transport. La cimenterie contribua à la croissance commerciale de la société BPD qui, grâce à ses participations dans le secteur textile ainsi que de l'énergie, devint un des plus grands groupes industriels du pays [CON 54], capable d'absorber la main-d'œuvre et de contribuer à apaiser le chômage qui dans l'après-guerre sévissait dans la nation.

\section{Le premier village industriel Bombrini Parodi Delfino}

Durant sa première phase d'activité, l'établissement chimique de Colleferro employait des ouvriers très spécialisés et formés à Avignana (Piémont) dans la fabrique de dynamite de la Société Anonyme Italienne Dynamite Nobel, et un nombre toujours plus élevé d'ingénieurs. À la fin de la période d'adaptation et de rodage de la production, l'usine s'ouvrit à la main-d'œuvre locale, formée de façon appropriée. Lentement, l'établissement commença à demander toujours plus de travailleurs, augmentant le niveau d'emploi de la région jusqu'à devenir un pôle d'attraction extrarégional. Les effets sur l'économie locale furent très élevés. La BPD donna un fort élan au développement de la zone grâce à la construction de nouveaux bâtiments et de nouvelles structures et d'habitations. Pour améliorer le lien entre les employés et leur lieu de travail et créer un sentiment d'appartenance, Parodi Delfino commanda en 1916 à l'architecte Michele Oddini, la construction d'un village pour les ouvriers [LEP 93] : le premier projet fut réalisé entièrement par un acteur privé sans l'intervention de l'État [ORL 11]. Un projet précurseur dont l'ingénieur espérait qu'il puisse devenir un modèle à imiter, capable de créer un espace de travail sans conflits sociaux et de former la classe ouvrière du futur. Le village ouvrier disposait de tous les services fondamentaux (hôpital, pharmacie, bureau de poste, école, crèches, etc.), mais aussi d'un cinéma, d'un théâtre, d'un hôtel, de restaurants et de bars [LEO 27 ${ }^{38}$. Il fournit aussi aux ouvriers une entreprise agricole avec des terrains que pouvaient cultiver les familles [ILV 27]. Le village était géré et administré par un gouverneur, un représentant de la société ayant des fonctions de contrôle, d'administration, de comptabilité et de trésorerie [LEO 27] ${ }^{39}$. Il fut terminé entre 1917 et 1918 . Il s'étendait sur une superficie d'environ 170.000 mètres carrés, sur lesquels 200 logements comprenant 700 pièces furent construits ${ }^{40}$.

La création du village, qui « suivait un véritable plan d'aménagement ${ }^{41}$, prévoyait l'attribution des habitations aux travailleurs sur des critères sociaux. Les unités aux architectures simples et linéaires étaient attribuées aux ouvriers et à leurs familles, tandis que les immeubles de luxe, c'est-àdire de type résidentiel, étaient destinés aux employés, aux diplômés et aux dirigeants. L'avancement de carrière garantissait le passage de l'unité habitation de base à la plus raffinée.

\footnotetext{
38 «Les œuvres d'assistance sociale créées par les industriels : Leopoldo Parodi Delfino créa le Village Moderne Industriel de Colleferro de Roma ", L'assistenza sociale nell'industria, a. 1, n 1, p. 12-19, 1927.

${ }^{39}$ Ivi, p. 15-16.

${ }^{40}$ ASCColleferro, BPD, 25 ans de vie de la B. P. D., Relazione del dottore Tito Benelli, Directeur de l'usine, octobre 1912-octobre 1937, p. 11. 
Avec le développement de l'établissement de Colleferro, le village fut agrandi. L'expansion de la « ville ouvrière » fut déléguée à l'ingénieur Riccardo Morandi qui planifia aussi la mairie, la Maison du Fascio, l'Église et les autres structures scolaires. Les logements dépendant de l'industrie, entre 1934 et 1936, comptaient plus de 400 unités.

Enfin, en 1938, un Institut pour l'hébergement et l'éducation des fils des victimes fut construit après le grand incendie qui toucha les établissements de Colleferro en janvier et qui provoqua la mort de nombreux travailleurs [ORL 11]. L'intention de Parodi était d'accueillir les orphelins et de les suivre jusqu'à qu'ils «terminent leur parcours d'écolier» [MAZ 80]. L'attention portée à ses ouvriers et à leurs familles apparaissait comme une priorité de l'entrepreneur qui se confirma de nouveau lors de cet accident. La construction de l'Institut pour les orphelins témoignait des préoccupations humaines de l'entrepreneur.

\section{Conclusion : Leopoldo Parodi Delfino, entrepreneur-savant}

Leopoldo Parodi Delfino fut une personnalité de référence des milieux économique et industriel de l'Italie de la première moitié du $\mathrm{XX}^{\mathrm{e}}$ siècle. Il incarna le modèle de l'entrepreneur schumpétérien, c'est-à-dire un personnage en mesure de conjuguer la recherche et l'invention pour les traduire en un processus d'innovation, un "pont » entre la science et l'entreprise, dans lequel l'entrepreneur s'appropriait l'invention technique qu'il introduisait dans l'activité productive pour la transformer rapidement en profit important [SCH 93]. Un homme capable de créer un nouveau cycle technologique et doué du courage nécessaire pour faire face continuellement à de nouveaux défis, grâce à son attention sur la diversification de la production et géographique des marchés. À ces capacités managériales, il ajouta une intense activité dans la recherche scientifique, dédiant aux expérimentations chimiques «les heures du jour et spécialement celles de la nuit que les occupations de l'Usine lui laissaient disponibles ${ }^{42}$. Parodi Delfino réalisa complètement la liaison entre l'industrie et la science, typique des règles schumpétériennes, devenant ainsi l'acteur et le créateur du progrès technique et économique. Un entrepreneur-savant qui de façon pionnière soumis la recherche à la création de nouveaux types de produits pour l'industrie pharmaceutique, l'agriculture et les usages domestiques. Il fit construire des laboratoires qui firent avancer la recherche : en 1924 il expérimenta la phthalide comme gélifiant, avec un dépôt de brevet; en 1927 il fut le premier à produire en Italie le TNT solidifié à un plus de $80,5^{\circ 43}$; en 1928 il expérimenta la nitration de pentaérythritol, de urotropine, mais aussi le traitement de la PETN (pentrite), du mètresol et de ses dérivés.

La recherche et les innovations ont également concerné, on l'a vu, le secteur des explosifs. A partir de 1930, l'usine BPD réalisa entièrement la production des cartouches et compléta ses installations pour produire du fulminate de mercure et de la solénite, utilisée dans les cartouches calibre 6,5 $\mathrm{mm}$ (chargées avec solénite) et calibre 13,2 $\mathrm{mm}$ (chargées avec la nouvelle poudre phtalide $)^{44}$. En 1932, en outre, la production des cartouches de calibre 20 et celle des douilles de canon commença. Entre 1935 et 1936, la technologie du secteur a permis la production de produits azotés utilisés pour le chargement des détonateurs militaires ${ }^{45}$. En 1937, la société Bombrini Parodi Delfino, après avoir été transformée en 1919 en société en commandite par actions, adopta le statut de société anonyme par actions. Pendant ces années, la production de Colleferro atteignit « le plus

\footnotetext{
42 Ivi, p. 14.

${ }^{43}$ Ivi, p. 10.

${ }^{44}$ Ibidem.

${ }^{45}$ Ivi, p. 11.
} 
haut niveau de performance ${ }^{46}$, ce qui satisfaisait les demandes de production du régime fasciste. Cela renforça la position industrielle de Parodi à l'intérieur du tissu économique national même si nous ne pouvons dire si ses activités étaient directement subordonnées au régime ou s'ils étaient les résultats d'un entrepreneur « libre ». Peu après la fin de la Seconde Guerre mondiale, le 3 Novembre 1945, Parodi Delfino meurt en laissant son activité à sa famille et à ses collaborateurs, mais aussi un très grand exemple d'esprit d'entreprise et d'ingéniosité.

\section{Références}

\section{Sources d'archives}

\section{Colleferro (Roma) :}

Archive Historique Mairie de Colleferro (ASCColleferro), Archive Bombrini-Parodi Delfino (BPD), Il centro industriale di Colleferro, MCMLI.

ASCColleferro, BPD, 25 années de vie de la B. P. D., Relazione del dottore Tito Benelli, Directeur de l'usine, octobre 1912octobre 1937.

ASCColleferro, BPD, Relazione del dottore Aldo Colajacono, Origini dello stabilimento BPD di Colleferro, 24 février 1966, p. 10.

\section{Genova :}

Archive Historique Ansaldo (ASA), Archive Parodi Delfino (APD), Acquedotto Pugliese.

ASA, Société Bombrini Parodi Delfino (SBPD), cc. 210, 1912-1936, Relation Leopoldo Parodi Delfino, juin 1912.

\section{Milano :}

Archive Historique Banca Intesa (ASI), Banca Commerciale Italiana (BCI), Bureau des Fiannces, Pratiques du Secrétatriat des Finances Milano, 14 Juin 1929.

ASI, BCI, Verbal du Conseil D’Administration, Vol. 6, p. 41, 22 octobre 1920.

ASI, BCI, Bureau des Finances, Pratiques du Secrétariat des finances, 21, 4, Milano, 14 juin 1929.

ASI, BCI, Bureau des Finances, Pratiques du Secrétariat des Finances, 21, 4, Extrait des délibérations Assemblée ordinaire et extraordinaire, 17 juin 1936.

\section{Roma :}

Archive Historique Ministère des Affaires Étrangères (ASMAE), Affaires Politiques, 1919-1930, Equateur, 1013, 2844, Programma per una penetrazione economica italiana nell'Equatore.

Archive Central de l’État, Épuration Sénat, Mémoires défensives, mémoire du 20 janvier 1947.

\section{Sources de presse}

Il Confederazione Generale del Lavoro (Confédération Générale du Travail), il Lazio, Roma, 1954.

Il Popolo d'Italia (Le Peuple d'Italie), 10 août 1939.

Regio Decreto Luogotenenziale (Décret Royal Législatif) du 10 avril 1919.

Senato del Regno (Sénat du Règne), Resoconti delle discussioni (Comptes-rendu des discussions), XXX legislatura (Législation), 1 della Camera dei Fasci e delle Corporazioni (de la Chambre des Faisceaux et des Corporations), Année 1940 (XVIII-XIX) Volume secondo (deuxième volume) p. 126, Roma, Tipografia del Senato (Imprimerie du Sénat), 1941 - XIX.

Senato della Repubblica (Sénat de la République), Relazione dell'Alta Corte di Giustizia per le sanzioni contro il fascismo, 22 février 1943. 


\section{Bibliographie}

[AET 28], AEta A., Molinari H., « Le grandi industrie chimiche. Gli acidi inorganici - solforico, cloridirico - fabbricazione, macchinari e impianti », L'ingegnere. Rivista tecnica del Sindacato nazionale fascista ingegneri, vol. II, p. 49 , 1928.

[AMA 11] Amatori F., Colli A., Storia d'impresa, Mondadori, Milano, 2011.

[BAR 86] BARONE G., Mezzogiorno e modernizzazione, Einaudi, Torino, 1986.

[BAR 18] BARUCCI P., Il corporativismo nell'Italia di Mussolini. Dal declino delle istituzioni liberali alla Costituzione repubblicana, Firenze University Press, 2018.

[BES 93] BESANA C., La prima industrializzazione della penisola tra arretratezza e squilibri (1896-1914), dans Zaninelli S. (a cura di), L'Ottocento economico italiano, Moduzzi, Bologna, 1993.

[CAN 97] CANDELA S., Florio Ignazio, junior, in Dizionario Biografico degli Italiani, vol. 48/1997, ad vocem.

[CAN 99] CANOSA R., Storia dell'epurazione in Italia, Baldini \& Castoldi, Milano, 1999.

[CAS 94] CASTRonovo V. (édité), Le origini, 1853-1882, Laterza, Roma-Bari, 1994.

[CAS 95] Castronovo V., Storia economica d'Italia. Dall'Ottocento ai giorni nostri, Einaudi, Torino, 1995.

[CAS 97] CAStronovo V. (édité), L’Ansaldo e la Grande Guerra 1915-1918, Laterza, Roma-Bari, 1997.

[DAM 80] Damiani C., Mussolini e gli Stati Uniti 1922-1935, Cappelli, Bologna, 1980.

[DAT 94] D'ATTORRE P.P., Studi sull'agricoltura italiana. Società rurale e modernizzazione, Annali, anno ventinovesimo 1993, Fondazione GianGiacomo Feltrinelli, Milano 1994.

[DEF 28] DE FAZI R., « Recensione Gli acidi inorganici - solforico, cloridrico - fabbricazione, macchinari e impianti », L’ingegnere. Rivista tecnica del Sindacato nazionale fascista ingegneri, vol. II, 1928, p. 547.

[DOR 98] DORIA M., L'imprenditoria industriale in Italia dall'Unità al «Miracolo economico». Capitani d'industria, padroni, innovatori, Giappichelli, Torino, 1998.

[FAT 16] FATANO R., La rivoluzione di Mussolini. La politica economica del fascismo tra corruzione ed innovazione, Milella, Lecce, 2016.

[GRO 31] GROTTARELLI F., Dinamite, in «Enciclopedia italiana Treccani», 1931, ad vocem.

[HER 96] Hertner P. (édité), Dai Bombrini ai Perrone, 1903-1914, Laterza, Roma-Bari, 1996.

[ILG 62] Il Gruppo industriale Bombrini Parodi-Delfino, Ed. B.P.D. Industriale Group, Milano, 1962.

[ILV 27] « Il Villaggio Industriale di Colleferro di Roma della Società Bombrini Parodi - Delfino », Il Dopolavoro, p. 24-31, 1927.

[LAC 46] La chimica e l'industria. Società chimica italiana, Associazione italiana di chimica, Federazione nazionale fascista degli industriali dei prodotti chimici, Anno XXVIII, Editrice di Chimica, Milano, 1946.

[LEO 27] «Le opere di assistenza sociale create dagli industriali: Leopoldo Parodi Delfino fonda il Villaggio Moderno Industriale di Colleferro di Roma », L'assistenza sociale nell'industria, a. 1, n 1, p. 13-19, 1927.

[LEP 93] LEPORE L., Lavoro di una comunità, Tipolitografia Ferrazza \& Bonelli, pp. 43, 110, 1993.

[LIZ 11] LIZZA G., «L'industria italiana della difesa nei 150 anni dell'Unità d'Italia », Studi e ricerche socio-territoriali, Napoli, 1, p. 83-98, 2011.

[MAR 12] MARSHALL A., Explosives: their manufacture, properties, tests and history, Blakiston, London, 1912.

[MAR 83] MARCElli C., RAIMOndo G., SCARnONe D., Fonti orali e didattica, Varigrafica Cappa, Colleferro, 1983.

[MAZ 80] Mazzocchi U., Colleferro, Ernesto Gremese Editore, Roma, 1980.

[MES 17] MeSsinA A., L'economia nello Stato totalitario fascista, Aracne, Ariccia, 2017.

[MOL 32] Molinari H., Grottanelli F., Cordova A., Esplosivi, in «Enciclopedia italiana Treccani», 1932, ad vocem.

[ORL 11] de ORLEans-Borbón A., Leopoldo Parodi Delfino, Talete, Roma, 2011.

[PEC 05] PECORARI P., L'Italia economica, Cedam, Milano, 2005.

[QUI 15] QUILIGOTTI G., « L’acquedotto pugliese a Genova », Altronovecento, Ambiente Tecnica Società. Rivista digitale fondata da Giorgio Nebbia, $\mathrm{n}^{\circ} 27,2015$.

[Ros 53] Rossi E., Lo Stato industriale, Laterza, Bari, 1953. 
[SAN 84] SANTARELLI E., «L'influsso dell'analisi schumpeteriana della funzione imprenditoriale e del credito nel pensiero economico italiano tra le due guerre », Giornale degli Economisti e Annali di Economia, 43, 7/8, p. 507-529, 1984.

[SCA 11] SCALERA I., I grandi imprenditori del XIX secolo, Cedam, Milano, 2011.

[SCH 11] SCHUMPETER JosePH A., Théorie de l'évolution économique, Dalloz, Paris, (1911) 1935.

[SAL 93] Salsano A., Joseph A. Schumpeter. L’imprenditore e la storia dell'impresa, Boringhieri, Torino, 1993.

[SOA 08] SOAVE P., La “scoperta geopolitica dell'Ecuador. Mire espansionistiche dell'Italia ed egemonia del dollaro 1919-1945, Franco Angeli, Milano, 2008.

[TON 13] TONIOLO G., La crescita economica italiana, 1861-2011, selon G. TONIOLO, L'Italia e l'economia mondiale dall'Unità a oggi, Marsilio, Venezia, p. 5-52, 2013.

[TOS 02] TOSCANO P., Le origini del capitalismo industriale nel Lazio, Edizioni dell’Università degli studi di Cassino, Cassino, 2002.

« Necrologio di Leopoldo Parodi-Delfino », La chimica e l'industria, Milano, n 1-2, p. 27, 1946.

\section{Sitographie :}

https://cittamorandiana.it/la-nascita-della-bpd/ (consulté le 11 juin 2020). 